\title{
DISPERSION POINTS FOR LINEAR SETS AND \\ APPROXIMATE MODULI FOR \\ SOME STOCHASTIC PROCESSES
}

\author{
BY \\ DONALD GEMAN ${ }^{1}$
}

\begin{abstract}
Let $\Gamma \in[0,1]$ be Lebesgue measurable; then $\Gamma$ has Lebesgue density 0 at the origin if and only if

$$
\int_{\Gamma} t^{-1} \Psi\left(t^{-1} \operatorname{meas}\{\Gamma \cap(0, t)\}\right) d t<\infty
$$

for some continuous, strictly increasing function $\Psi(t)(0<t<1)$ with $\Psi(0)=0$. This result is applied to the local growth of certain Gaussian (and other) proceses $\left\{X_{t}, t>0\right\}$ as follows: we find continuous, increasing functions $\phi(t)$ and $\eta(t)(t>0)$ such that, with probability one, the set $\{t$ : $\left.\eta(t)<\left|X_{t}-X_{0}\right|<\phi(t)\right\}$ has density 1 at the origin.
\end{abstract}

1. Introduction. This paper is about points of dispersion (i.e., zero Lebesgue density) for sets of real numbers with applications to finding approximate upper and lower moduli for Brownian motion and other processes. By such moduli (say at the origin) for a process $\left\{X_{t}(\omega), t \geqslant 0\right\}$, I mean continuous, nondecreasing functions $\phi$ and $\eta(\phi(0)=\eta(0)=0)$ such that, with probability one, each of the sets

$$
\left\{t:\left|X_{t}(\omega)-X_{0}(\omega)\right|>\phi(t)\right\}, \quad\left\{t:\left|X_{t}(\omega)-X_{0}(\omega)\right|<\eta(t)\right\}
$$

has $t=0$ as a point of dispersion. This is a property of the trajectories and could as well be defined for a fixed function.

Here, first, are the analytical results. Let $\Gamma$ be a bounded, Lebesgue measurable set of positive real numbers. I will write " $0 \in \mathrm{dp}(\Gamma)$ " if $t=0$ is a point of dispersion for $\Gamma$ :

$$
\lim _{\varepsilon \downarrow 0} \varepsilon^{-1} m(\Gamma \cap(0, \varepsilon))=0
$$

( $m$ denotes Lebesgue measure).

Received by the editors August 29, 1978.

AMS (MOS) subject classifications (1970). Primary 28A10, 60G17; Secondary 26A15, 60G15. Key words and phrases. Lebesgue density, approximate upper (lower) modulus, approximate continuity, Brownian motion, Gaussian process, scale-invariance.

'This work was supported by National Science Foundation grant MCS 76-06599. 
THEOREM 1. In order that $0 \in \mathrm{dp}(\Gamma)$, it is necessary and sufficient that

$$
\int_{\Gamma} \frac{1}{t} \Psi\left(\frac{m(\Gamma \cap(0, t))}{t}\right) d t<\infty
$$

for some continuous, strictly increasing function $\Psi(t)(0 \leqslant t \leqslant 1)$ with $\Psi(0)=$ 0.

The sufficiency of (3) when $\Psi=$ constant is almost immediate. Let $I_{\Gamma}(t)$ be the indicator function of $\Gamma$. Then

$$
\frac{m(\Gamma \cap(0, \varepsilon))}{\varepsilon}=\frac{1}{\varepsilon} \int_{0}^{\varepsilon} I_{\Gamma}(t) d t<\int_{0}^{\varepsilon} \frac{1}{t} I_{\Gamma}(t) d t,
$$

and consequently,

$$
\int_{\Gamma} \frac{1}{t} d t<\infty \Rightarrow 0 \in \mathrm{dp}(\Gamma) .
$$

An apparent improvement of (4) would be to replace the integrand $t^{-1}$ by $t^{-1} \Lambda(t)$ where, say, $\Lambda(0)=0, \Lambda$ increases, and $t^{-1} \Lambda(t)$ decreases. However, given any such $\Lambda$ there is a set $\Gamma$ which invalidates (4): see the Appendix. In effect, what Theorem 1 does is to allow $\Lambda$ to depend on $\Gamma$.

The utility of Theorem 1 for probability theory is this: let $\Gamma(\omega)$ be a random set; it is often difficult to prove that the limit in (2) exists, and hence to prove directly that $0 \in \operatorname{dp}(\Gamma(\omega))$ a.s. On the other hand, it is often relatively easy to prove that the (random) quantity in (3) has a finite expected value for suitable $\Psi$ 's, and hence is finite a.s.

The proof of Theorem 1 is deferred to the Appendix; the necessity part is very easy, whereas the sufficiency part is rather involved. Actually, the same proof yields a more general result, namely:

Let $0 \leqslant f(t) \leqslant 1(0 \leqslant t \leqslant 1)$ be Lebesgue measurable; then

$$
\lim _{\varepsilon \downarrow 0} \frac{1}{\varepsilon} \int_{0}^{\varepsilon} f(t) d t=0
$$

if and only if

$$
\int_{0}^{1} \frac{f(t)}{t} \Psi\left[\frac{1}{t} \int_{0}^{t} f(x) d x\right] d t<\infty
$$

for some $\Psi$ as described in Theorem 1 .

Here is a short proof of the sufficiency part in the special case when $\Psi$ is convex. This case covers all the applications below in which the $\Psi$ 's that are used are powers: $\Psi(t)=t^{n}, n \geqslant 1$. I wish to thank the referee for suggesting this approach.

Proof that $\left(3^{\prime}\right) \Rightarrow\left(2^{\prime}\right)$ for $\Psi$ convex. Since $\left(3^{\prime}\right)$ holds, we have

$$
\lim _{\varepsilon \downarrow 0} \int_{0}^{e} \frac{f(t)}{t} \Psi\left[\frac{1}{t} \int_{0}^{t} f(x) d x\right] d t=0 .
$$


After changing variables, the integral above is

$$
\begin{aligned}
\int_{0}^{1} \frac{f(t \varepsilon)}{t} \Psi\left(\frac{1}{t \varepsilon} \int_{0}^{t \varepsilon} f(x) d x\right) d t & \geqslant \int_{0}^{1} f(t \varepsilon) \Psi\left(\frac{1}{t \varepsilon} \int_{0}^{t \varepsilon} f(x) d x\right) d t \\
& \geqslant \int_{0}^{1} \Psi\left(\frac{f(t \varepsilon)}{t \varepsilon} \int_{0}^{t \varepsilon} f(x) d x\right) d t
\end{aligned}
$$

(since $\Psi$ is convex and $0 \leqslant f \leqslant 1$ )

$$
\geqslant \Psi\left(\int_{0}^{1} \frac{f(t \varepsilon)}{t \varepsilon} \int_{0}^{t \varepsilon} f(x) d x d t\right)
$$

(by Jensen's inequality)

$$
\begin{aligned}
& =\Psi\left(\int_{0}^{1} \frac{f(t \varepsilon)}{t} \int_{0}^{t} f(x \varepsilon) d x d t\right) \geqslant \Psi\left(\int_{0}^{1} \int_{0}^{t} f(x \varepsilon) f(t \varepsilon) d x d t\right) \\
& =\Psi\left(\frac{1}{2}\left(\int_{0}^{1} f(t \varepsilon) d t\right)^{2}\right)=\Psi\left(\frac{1}{2}\left(\frac{1}{\varepsilon} \int_{0}^{\varepsilon} f(t) d t\right)^{2}\right) .
\end{aligned}
$$

Hence,

$$
\lim _{\varepsilon \downarrow 0} \Psi\left(\frac{1}{\varepsilon} \int_{0}^{\varepsilon} f(t) d t\right)=0
$$

from which (2') follows, which completes the proof.

2. Approximate moduli. Let $X=\left\{X_{t}(\omega), t \geqslant 0\right\}$ be a stochastic process; the probability measure is denoted $\mathbf{P}$ and the integration with $d \mathbf{P}$ is denoted $\mathbf{E}$. Let $\bar{\Gamma}(\phi ; \omega)$ and $\bar{\Gamma}(\eta ; \omega)$ stand for the first and second sets in (1). Convention has it that $\phi$ is an "upper function" or "modulus of continuity" at $t=0$ if, with probability one, the set $\bar{\Gamma}(\phi ; \omega)$ does not accumulate at $t=0 ; \phi$ is a "lower function" if $\bar{\Gamma}(\phi ; \omega)$ does accumulate there a.s. This distinction is uninteresting for $\Gamma(\eta ; \omega)$ because, for most of the important (nondifferentiable) processes, the set $\left\{t: X_{t}(\omega)=X_{0}(\omega)\right\} \subset \underline{\Gamma}(\eta ; \omega)$ will itself have $t=0$ as a limit point.

I will call $\phi$ an approximate upper modulus (for $X$ at $t=0$ ) if $0 \in$ $\operatorname{dp}(\bar{\Gamma}(\phi ; \omega))$ a.s. and $\eta$ an approximate lower modulus $($ for $X$ at $t=0)$ if $0 \in \mathrm{dp}(\underline{T}(\eta ; \omega))$ a.s. (See $\$ 3$ for an interpretation via limit points.) To insure that $\bar{\Gamma}$ and $\underline{\Gamma}$ are Lebesgue measurable, let us assume that $X$ is jointly measurable with respect to the Lebesgue $\sigma$-field and the events in our probability space. Any measurable version will do: separability is irrelevant and altering the sample paths on a Lebesgue null set makes no difference. In fact, even measurability may be superfluous: the definition of dispersion point (and hence of $\phi$ and $\eta$ ) can be formulated with Lebesgue outer measure and it is then possible, although I have not been able to determine whether it is so, that some of the probabilistic results depend only on the law of $X$, i.e. are valid for every representation of the process. 
3. Digression. There is another way to think of an approximate upper modulus, just for a real function $F: t=0$ is not a limit point for $\{t$ : $|F(t)-F(0)|>\phi(t)\}$ relative to the density topology. This topology was introduced in [6]; see also [11] for some connections with probability theory. The open sets are those which are Lebesgue measurable and have density 1 at each of their points. $F$ is then a "Borel function" if and only if $F$ is Lebesgue measurable, and hence if and only if $F$ is approximately continuous $m$-a.e. Recall that $F$ is approximately continuous at $t$ if

$$
\lim _{\substack{s \rightarrow t \\ s \in A}} F(s)=F(t)
$$

for some set $A$ with density 1 at $t$. Thus, an approximate upper modulus always serves as a "modulus of approximate continuity" (at $t=0$ ) whether or not $F$ is actually continuous there.

4. An application of (4). With $X$ and $\bar{\Gamma}(\phi ; \omega)$ as in $\S 2$,

$$
\mathbf{E} \int_{\Gamma(\phi ; \omega)} \frac{1}{t} d t=\int_{0}^{1} \frac{1}{t} \mathbf{P}\left\{\left|X_{t}-X_{0}\right|>\phi(t)\right\} d t,
$$

which can be shown to be finite under a variety of conditions on $\mathbf{P}\left\{X_{t}-X_{0}\right.$ $\in d x\}$. For example, let $X$ be Gaussian with zero means and incremental variance $\sigma^{2}(t)=\mathbf{E}\left(X_{t}-X_{0}\right)^{2}, t \geqslant 0$. Then the expression in (5) is

$$
2 \int_{0}^{1} \frac{1}{t}\left(1-N\left(\frac{\phi(t)}{\sigma(t)}\right)\right) d t
$$

where $N$ is the standard normal distribution function. Put $h=\phi / \sigma$ and assume $h \uparrow+\infty$ as $t \downarrow 0$. Then (6) is finite if and only if, for some $\varepsilon>0$,

$$
\int_{0}^{\varepsilon} \frac{1}{\operatorname{th}(t)} \exp \left(-\frac{h^{2}(t)}{2}\right) d t<\infty
$$

In particular, the function

$$
\phi(t)=\sigma(t)((2+\varepsilon) \log |\log t|)^{1 / 2} \quad(\varepsilon>0)
$$

is an approximate upper modulus for any Gaussian process-continuous or not-for which $\sigma$ is continuous and $\phi \downarrow 0$ as $t \downarrow 0$.

For Brownian motion, Kolmogorov's test [7, p. 33] states that $\phi$ is an upper function if

$$
\int_{0}^{\varepsilon} \frac{h(t)}{t} \exp \left(-\frac{h^{2}(t)}{2}\right) d t<\infty \quad(\text { some } \varepsilon>0)
$$

and $\phi$ is a lower function if the integral diverges. Evidently, we can choose $h$ so that (7) holds whereas (8) fails.

The motivation for improving (4) was to show that $\phi(t)=(2 t \log |\log t|)^{1 / 2}$ is an approximate upper modulus for Brownian motion and other Gaussian processes. For this $\phi$, both (7) and (8) fail. 
5. Example. Theorems 2, 3, and 4 below identify $\phi$ 's and $\eta$ 's (in varying degrees of explicitness) for classes of processes which I call "scale-invariant" (see \$6). Here is perhaps the best illustration I have of these results.

Let $X^{\alpha}$ be the Gaussian process with zero mean and covariance

$$
R(s, t)=\mathbf{E} X_{t} X_{s}=\frac{1}{2}\left\{t^{2 \alpha}+s^{2 \alpha}-|t-s|^{2 \alpha}\right\},
$$

$0<\alpha<1 . X^{1 / 2}$ is ordinary Brownian motion. Then $\phi$ and $\eta$ may be selected as follows (see $\S \S 7,8)$ :

$$
\begin{aligned}
& \phi(t)=t^{\alpha}(2 \log |\log t|)^{1 / 2}, \\
& \eta(t)=t^{\alpha}|\log t|^{-\delta} \text { for any } \delta>0 .
\end{aligned}
$$

Note. It is known (see, e.g., Marcus [9, III:2.4]) that there is a constant $0<C<\infty$ such that $C \phi(t)$ is a local modulus of continuity for any separable version of $X^{\alpha}$.

6. Scale-invariant processes. Let $X$ be as in $\S 2$ and suppose that $X_{0} \equiv 0$; otherwise just subtract $X_{0}$ from $X$. Suppose also that $\mathbf{E} X_{t} \equiv 0$ and $\sigma^{2}(t) \equiv$ $\mathbf{E} X_{t}^{2}$ is finite, positive, and continuous for all $t>0$. I will call $X$ scale-invariant if, for each $c>0$, the distribution of the process

$$
\left\{\frac{X_{c t}}{\sigma(c t)}\right\}, \quad t>0,
$$

does not depend on $c$. In particular, the correlation coefficient

$$
\rho\left(X_{s}, X_{t}\right)=\frac{\mathbf{E} X_{s} X_{t}}{\sigma(s) \sigma(t)}
$$

depends only on the ratio $s / t$, say $\rho\left(X_{s}, X_{t}\right)=\xi(s / t)$, where $\xi(1)=1$ and $\xi(r)=\xi(1 / r), r>0$. The condition that $\xi$ exist constitutes a "wide sense" version of scale-invariance and the two notions agree in the Gaussian case.

A related class of processes has been variously called "scale-invariant" or "self-similar": $X_{0} \equiv 0$ and there exists an $H>0$ such that, for each $c>0$, the two processes $\left\{X_{c t}\right\}$ and $\left\{c^{H} X_{t}\right\}, t>0$, have the same distribution. It is easy to check (assuming second moments) that $X$ belongs to this class if and only if $X$ is scale-invariant and $\sigma(t)=\sigma(1) \cdot t^{H}$ for some $H>0$.

7. Some Gaussian cases: upper moduli. Here and in the following section, we suppose that $X$ is Gaussian and scale-invariant. The proof of Theorem 2 below boils down to the behavior of $\xi(r)$ near $r=1$. In this section, we will assume that:

(i) $\xi(r)<1$ for $r<1$ (equivalently, $\rho\left(X_{s}, X_{t}\right)<1$ for all $s \neq t$ );

(ii) $\xi(r)$ is increasing on $\left[r_{0}, 1\right]$ for some $r_{0}<1$;

(iii) $\lim _{r \uparrow 1} \xi(r)=1$. 
Let $\hat{\xi}$ denote the inverse of $\xi$ :

$$
\hat{\xi}(y)=\inf \{r: \xi(r)>y\}, \quad \xi\left(r_{0}\right)<y<1 .
$$

Let $h(t)$ be continuous and decreasing on $(0, \delta]$ with $h\left(0^{+}\right)=\infty$ and inverse $\hat{h}$, and suppose that $\phi(t) \equiv \sigma(t) h(t) \downarrow 0$ as $t \downarrow 0$.

THEOREM 2. Suppose that

$$
1-\hat{\xi}(y) \sim(1-y)^{\beta} L(1-y) \text { as } y \uparrow 1
$$

where $\beta>0$ and $L$ is slowly varying at the origin. Suppose also that

$$
\int_{h(\delta)}^{\infty}|\log \hat{h}(x)| e^{-x^{2} / 2} x^{-2 \beta} L\left(x^{-2}\right) d x<\infty .
$$

Then $\phi(t)$ is an approximate upper modulus. In particular,

$$
\phi(t)=\sigma(t)(2 \log |\log t|)^{1 / 2}
$$

is an approximate upper modulus whenever $1-\xi(r) \sim$ const $\times(1-r)^{\gamma}$ for some $\gamma<2$.

As an example, consider the class of processes $X^{\alpha}, 0<\alpha<1$, described in $\S 5$. Here $\sigma^{2}(t)=t^{2 \alpha}$ and

$$
\xi(r)=\frac{1}{2}\left\{\frac{1+r^{2 \alpha}-(1-r)^{2 \alpha}}{r^{\alpha}}\right\}, \quad 0 \leqslant r \leqslant 1 .
$$

Moreover, (i)-(iii) hold (details aside) and by expanding $r^{\alpha}$ and $r^{2 \alpha}$ in second-order Taylor series about $r=1$ we find that

$$
\begin{aligned}
1-\xi(r) & =r^{-\alpha}\left(\frac{1}{2}(1-r)^{2 \alpha}+O\left((1-r)^{2}\right)\right) \\
& \sim \frac{1}{2}(1-r)^{2 \alpha} \text { as } r \uparrow 1 .
\end{aligned}
$$

Consequently, (9) holds with $\beta=1 / 2 \alpha$ and $L \equiv 2^{1 / 2 \alpha}$.

Proof. According to Theorem 1 with $\Psi(t)=t$, and writing $\Gamma(\omega)$ for $\bar{\Gamma}(\phi ; \omega)$, it will suffice to prove that

$$
\int_{0}^{\delta} \frac{1}{t} I_{\Gamma(\omega)}(t)\left(\frac{m(\Gamma(\omega) \cap(0, t))}{t}\right) d t<\infty \quad \text { a.s. }
$$

By Fubini's theorem, the expected value of the random variable in (11) is

$$
\begin{aligned}
\int_{0}^{\delta} t^{-2} \int_{0}^{t} \mathbf{P} & \left\{X_{s}>\phi(s), X_{t}>\phi(t)\right\} d s d t \\
& =\int_{0}^{\delta} t^{-1} \int_{0}^{1} \mathbf{P}\left\{X_{r t}>\phi(r t), X_{t}>\phi(t)\right\} d r d t \quad\left(r=\frac{s}{t}\right) \\
& \left.<\int_{0}^{\delta} t^{-1} \int_{0}^{1} \mathbf{P}\left\{\frac{X_{r t}}{\sigma(r t)}>h(t), \frac{X_{t}}{\sigma(t)}>h(t)\right\} d r d t \quad \text { (since } h \downarrow\right) \\
& =\int_{0}^{\delta} t^{-1} \int_{0}^{1} \int_{h(t)}^{\infty} \int_{h(t)}^{\infty} p(x, y ; \xi(r)) d x d y d r d t,
\end{aligned}
$$


where $p(x, y ; \lambda)$ is the bivariate normal density with zero means, unit variances, and covariance $\lambda$. Write the last expression above as

$$
\int_{0}^{1} K(\xi(r)) d r
$$

where

$$
\begin{aligned}
K(\lambda) & =\int_{0}^{\delta} t^{-1} \int_{h(t)}^{\infty} \int_{h(t)}^{\infty} p(x, y ; \lambda) d x d y d t \\
& =\int_{c}^{\infty} \int_{c}^{\infty}\left(\int_{0}^{\delta} t^{-1} I_{(0, x)}(h(t)) I_{(0, y)}(h(t)) d t\right) p(x, y ; \lambda) d x d y \quad \quad(c=h(\delta)) \\
& =2 \int_{c}^{\infty} \int_{x}^{\infty}\left(\int_{\hat{h}(x)}^{\delta} \frac{d t}{t}\right) p(x, y ; \lambda) d y d x \\
& \leqslant \text { const } \times \int_{c}^{\infty}|\log \hat{h}(x)| \int_{x}^{\infty} p(x, y ; \lambda) d y d x .
\end{aligned}
$$

For each $t$ fixed, the function

$$
\lambda \rightarrow \int_{h(t)}^{\infty} \int_{h(t)}^{\infty} p(x, y ; \lambda) d x d y, \quad-1<\lambda<1,
$$

is nondecreasing. It then follows from (i)-(iii) that

$$
\int_{0}^{1} K(\xi(r)) d r \leqslant \text { const } \times \int_{r_{0}}^{1} K(\xi(r)) d r .
$$

In other words, the only singularity in (12) occurs at the upper limit of integration.

If $\hat{\xi}$ has a bounded derivative (so $\beta=1$ as in the Brownian case) there is a shortcut in the rest of the proof that seems worth mentioning. Let $d \hat{\xi} / d y<D$ $<\infty$ for $\xi\left(r_{0}\right) \leqslant y \leqslant 1$ and take $\xi\left(r_{0}\right)>0$. Then

$$
\begin{aligned}
\int_{r_{0}}^{1} K(\xi(r)) d r & =\int_{\xi\left(r_{0}\right)}^{1} K(\lambda) \frac{d \hat{\xi}}{d \lambda} d \lambda \leqslant D \int_{0}^{1} K(\lambda) d \lambda \\
& =\text { const } \times \int_{c}^{\infty}|\log \hat{h}(x)| \int_{x}^{\infty} \int_{0}^{1} p(x, y ; \lambda) d \lambda d y d x .
\end{aligned}
$$

A fairly well-known identity (see $[2$, p. 21]) is

$$
\int_{0}^{1} p(x, y ; \lambda) d \lambda=N(x \wedge y)-N(x) N(y) ;
$$

it results from the equation

$$
\frac{\partial}{\partial \lambda} p(x, y ; \lambda)=\frac{\partial^{2}}{\partial x \partial y} p(x, y ; \lambda)
$$


Hence,

$$
\begin{aligned}
\int_{r_{0}}^{1} K(\xi(r)) d r & \leqslant \text { const } \times \int_{c}^{\infty}|\log \hat{h}(x)| \int_{x}^{\infty} N(x)(1-N(y)) d y d x \\
& \leqslant \text { const } \times \int_{c}^{\infty}|\log \hat{h}(x)| e^{-x^{2} / 2} x^{-2} d x .
\end{aligned}
$$

The last inequality comes about because $1-N(y) \leqslant y^{-1} n(y)(n(y)=$ $\left.(2 \pi)^{-1 / 2} \exp \left(-y^{2} / 2\right)\right)$ so that

$$
\int_{x}^{\infty}(1-N(y)) d y<\int_{x}^{\infty} y^{-1} n(y) d y \leqslant x^{-1}(1-N(x)) \leqslant x^{-2} n(x) .
$$

Hence by (10) the proof is complete.

Going back to the general case, we must show that

$$
\int_{c}^{\infty}|\log \hat{h}(x)| \int_{r_{0}}^{1} \int_{x}^{\infty} p(x, y ; \xi(r)) d y d r d x<\infty .
$$

To simplify matters, suppose $r_{0}=\xi\left(r_{0}\right)=0$. An easy computation yields

$$
\begin{aligned}
\int_{x}^{\infty} p(x, y ; \xi(r)) d y & =n(x)\left(1-N\left(x\left\{\frac{1-\xi(r)}{1+\xi(r)}\right\}^{1 / 2}\right)\right) \\
& \leqslant n(x)\left(1-N\left(x\left\{\frac{1-\xi(r)}{2}\right\}^{1 / 2}\right)\right) .
\end{aligned}
$$

Now,

$$
\begin{aligned}
& \int_{0}^{1} 1-N\left(x\left\{\frac{1-\xi(r)}{2}\right\}^{1 / 2}\right) d r \\
& =1-n\left(\frac{x}{\sqrt{2}}\right)+\int_{0}^{1} \int_{(x / \sqrt{2})(1-\xi(r))^{1 / 2}}^{x / \sqrt{2}} n(w) d w d r
\end{aligned}
$$

The first term above is of smaller order than $x^{-2 \beta} L\left(x^{-2}\right)$ (as $\left.x \rightarrow \infty\right)$ and hence can be ignored. The second term equals

$$
\begin{aligned}
\int_{0}^{x / \sqrt{2}} n(w) & m\left\{0 \leqslant r \leqslant 1: x(1-\xi(r))^{1 / 2} \leqslant w \sqrt{2}\right\} d w \\
= & \int_{0}^{x / \sqrt{2}} n(w)\left(1-\hat{\xi}\left(1-2\left(\frac{w}{x}\right)^{2}\right)\right) d w \\
= & \frac{x}{4 \sqrt{\pi}} \int_{0}^{1} e^{-y\left(x^{2} / 4\right)}(1-\hat{\xi}(1-y)) y^{-1 / 2} d y \quad\left(y=2\left(\frac{w}{x}\right)^{2}\right) \\
= & \frac{x}{4 \sqrt{\pi}} J\left(\frac{x^{2}}{4}\right),
\end{aligned}
$$

where $J$ is the Laplace transform of the measure

$$
U(d y)=I_{[0,1]}(y)(1-\hat{\xi}(1-y)) y^{-1 / 2} d y \text {. }
$$


From (9), $1-\hat{\xi}(1-y) \sim y^{\beta} L(y)$ as $y \downarrow 0$, which implies that $U\{[0, y]\} \sim$ const $\times y^{\beta+1 / 2} L(y)$ as $y \downarrow 0$, which in turn implies (see e.g. [3, p. 422]) that

$$
J(\lambda) \sim \text { const } \times \lambda^{-(\beta+1 / 2)} L\left(\lambda^{-1}\right) \text { as } \lambda \rightarrow \infty .
$$

To conclude the proof, substitute $\lambda=x^{2} / 4$; then (10) implies (13).

8. Some Gaussian cases: lower moduli. For each integer $n \geqslant 1$ and vector $\mathbf{s}=\left(s_{1}, \ldots, s_{n}\right)$ with distinct components, let $D_{n}(\mathbf{s})$ denote the determinant of the covariance matrix of $\left(X_{s_{1}}, \ldots, X_{s_{n}}\right)$.

THEOREM 3. Suppose that

$$
\int_{[0,1]^{n}}\left(D_{n}(\mathrm{~s})\right)^{-1 / 2} d \mathrm{~s}<\infty
$$

for some $n \geqslant 1$. Then

$$
\eta(t) \equiv \sigma(t)|\log t|^{-a} \quad(a>1 / n)
$$

is an approximate lower modulus.

Condition $\left(D_{n}\right)$ occurs repeatedly in the analysis of the sample paths of Gaussian processes and, in particular, in the study of local times. Generally it is difficult to check $\left(D_{n}\right)$ "by hand". An exception is the Brownian case: Let $s_{1}^{*}, \ldots, s_{n}^{*}$ be the $s_{i}$ 's in ascending order; then

$$
D_{n}(\mathrm{~s})=s_{1}^{*} \prod_{k=2}^{n}\left(s_{k}^{*}-s_{k-1}^{*}\right)
$$

and it follows easily that $\left(D_{n}\right)$ holds for every $n \geqslant 1$.

Perhaps the best way of dealing in general with the condition $\left(D_{n}\right)$ involves the concept of local nondeterminism, which was introduced by S. Berman [1] in connection with Gaussian local times. For the exact definition as well as the proof of the following statement, consult the survey paper [4] where all these matters are discussed in detail.

Suppose $X$ is locally nondeterministic and

$$
\sup _{0<t<1} \int_{0}^{1}\left(\mathbf{E}\left(X_{s}-X_{t}\right)^{2}\right)^{-1 / 2} d s<\infty .
$$

Then $\left(D_{n}\right)$ holds for every $n$.

We note that (14) is satisfied whenever

$$
\mathbf{E}\left(X_{s}-X_{t}\right)^{2} \sim \text { const } \times|s-t|^{2 \alpha} \quad(0<\alpha<1) .
$$

Proof of Theorem 3. $\left(D_{n}\right)$ implies that

$$
\int_{0}^{b} \cdots \int_{0}^{b}\left(D_{n}\left(s_{1}, \ldots, s_{n-1}, b\right)\right)^{-1 / 2} d s_{1} \cdots d s_{n-1}<\infty
$$


for $m$-a.e. $b \in(0,1)$. Fix such $a b$ and consider

$$
\mathrm{E} \int_{0}^{b} \frac{1}{t} I_{\Gamma(\omega)} \Psi\left(\frac{m(\Gamma(\omega) \cap(0, t))}{t}\right) d t
$$

with $\Psi(t)=t^{n-1}, \Gamma(\omega)=\underline{\Gamma}(\eta ; \omega)$. This expectation is

$$
\begin{aligned}
& \int_{0}^{b} t^{-n} \int_{0}^{t} \cdots \int_{0}^{t} \mathbf{P}\left\{\left|X_{s_{1}}\right|<\eta\left(s_{1}\right), \ldots,\left|X_{s_{n-1}}\right|<\eta\left(s_{n-1}\right),\right. \\
&\left.\left|X_{t}\right|<\eta(t)\right\} d s_{1} \cdots d s_{n-1} d t .
\end{aligned}
$$

Make the change of variables $t \rightarrow b t, s_{i} \rightarrow s_{i} t, 1<i<n$. It then follows (using the monotonicity of $|\log t|$ ) that the expression in (15) is finite if the following expression is finite:

$$
\begin{aligned}
\int_{0}^{1} t^{-1} \int_{0}^{b} \cdots \int_{0}^{b} \mathbf{P}\left\{\max _{1<i<n-1} \frac{\left|X_{s_{i}}\right|}{\sigma\left(s_{i} t\right)}<|\log t|^{-a},\right. \\
\left.\qquad \frac{\left|X_{b t}\right|}{\sigma(b t)}<|\log t|^{-a}\right\} d s_{1} \cdots d s_{n-1} d t .
\end{aligned}
$$

By scale-invariance, the probability in (16) is

$$
\mathbf{P}\left\{\max _{1<i<n-1} \frac{\left|X_{s_{i}}\right|}{\sigma\left(s_{i}\right)}<|\log t|^{-a}, \frac{\left|X_{b}\right|}{\sigma(b)}<|\log t|^{-a}\right\}
$$

which is dominated by

$$
\sigma(b) \prod_{i=1}^{n-1} \sigma\left(s_{i}\right)\left(2|\log t|^{-a}\right)^{n}\left(D_{n}\left(s_{1}, \ldots, s_{n-1}, b\right)\right)^{-1 / 2}
$$

because the joint density of $\left(X_{s_{1}}, \ldots, X_{s_{1}-1}, X_{b}\right)$ is bounded by its value at the origin, namely $\left(D_{n}\left(s_{1}, \ldots, s_{n-1} b\right)\right)^{-1 / 2}$. Thus, the integral in (16) is dominated by a

$$
\text { const } \times \int_{0}^{1} \frac{d t}{t|\log t|^{n a}} \int_{0}^{b} \cdots \int_{0}^{b}\left(D_{n}\left(s_{1}, \ldots, s_{n-1}, b\right)\right)^{-1 / 2} d s_{1} \cdots d s_{n-1} \text {, }
$$

which is finite.

9. Extensions to non-Gaussian processes. Let $X$ be scale-invariant, though not necessarily Gaussian. The following theorem is quite apparent if one looks over the proofs of Theorems 2 and 3 to see which arguments rely on scale-invariance alone, taking $\Psi(t)=t^{n}$ and computing the outer " $d t$ " integral first (as in Theorem 2).

Let $h$ decrease for $0<t \leqslant \delta$, with $h\left(0^{+}\right)=+\infty$ and inverse $\hat{h}$; let $g$ increase for $0<t<\delta$, with $g(0)=0$ and inverse $\hat{g}$. In addition, suppose that 
$\phi(t) \equiv \sigma(t) h(t)$ and $\eta(t) \equiv \sigma(t) g(t)$ are continuous for $0<t<\delta$ and $\phi(t), \eta(t) \downarrow 0$ as $t \downarrow 0$.

Define

$$
\begin{aligned}
& H_{n}\left(x_{1}, \ldots, x_{n}\right)= \begin{cases}\max \left\{\hat{h}\left(\left|x_{1}\right|\right), \ldots, \hat{h}\left(\left|x_{n}\right|\right)\right\}, & \left|x_{i}\right|>h(\delta) \forall i, \\
1, & \text { otherwise; }\end{cases} \\
& G_{n}\left(x_{1}, \ldots, x_{n}\right)= \begin{cases}\max \left\{\hat{g}\left(\left|x_{1}\right|\right), \ldots, \hat{g}\left(\left|x_{n}\right|\right)\right\}, & \left|x_{i}\right|>g(\delta) \forall i, \\
1, & \text { otherwise. }\end{cases}
\end{aligned}
$$

THEOREM 4. $\phi$ is an approximate upper modulus if

$$
-\int_{0}^{1} \cdots \int_{0}^{1} \mathbf{E} \log H_{n}\left(\frac{X_{s_{1}}}{\sigma\left(s_{1}\right)}, \ldots, \frac{X_{s_{n}}}{\sigma\left(s_{n}\right)}\right) d s_{1} \cdots d s_{n}<\infty,
$$

and $\eta$ is an approximate lower modulus if

$$
-\int_{0}^{1} \cdots \int_{0}^{1} \mathbf{E} \log G_{n}\left(\frac{X_{s_{1}}}{\sigma\left(s_{1}\right)}, \ldots, \frac{X_{s_{n}}}{\sigma\left(s_{n}\right)}\right) d s_{1} \cdots d s_{n}<\infty .
$$

As in the previous section, the expression in (18) is readily bounded if we assume that the distribution of the random vector $X_{s_{1}} / \sigma\left(s_{1}\right), \ldots, X_{s_{n}} / \sigma\left(s_{n}\right)$ is well-behaved near the origin (in $\mathbf{R}^{n}$ ). Specifically, suppose that for each $\left(s_{1}, \ldots, s_{n}\right)$ in $[0,1]$ (with distinct $\left.s_{i}\right)$ the vector above has a density $p_{n}\left(\mathbf{x} ; s_{1}, \ldots, s_{n}\right), \mathbf{x} \in \mathbf{R}^{n}$, such that

$$
\int_{0}^{1} \cdots \int_{0}^{1} \sup _{|\mathbf{x}|<\varepsilon} p_{n}\left(\mathbf{x} ; s_{1}, \ldots, s_{n}\right) d s_{1} \cdots d s_{n}<\infty
$$

for some $\varepsilon>0$. Then (18) holds for $g(t)=|\log t|^{-a}, a>1 / n$.

10. Some related work. Several other papers have related ideas. First, there is a tenuous but interesting connection with a result of Strassen's [10]: let $S_{n}$ be the sum of the first $n$ random variables of an i.i.d. sequence with mean 0 and variance 1 ; then

$$
\limsup _{n \rightarrow \infty} \nu_{n}=1-\exp \left\{-4\left(\frac{1}{c^{2}}-1\right)\right\} \text { a.s., }
$$

where $\nu_{n}$ is the proportion of the first $n$ positive integers for which $S_{i}>$ $c(2 i \log \log i)^{1 / 2}, 1 \leqslant i \leqslant n(0 \leqslant c \leqslant 1)$. Choosing $c=1$ yields $\nu_{n} \rightarrow 0$ a.s., which is reminiscent of our result that, for Brownian motion,

$$
\varepsilon^{-1} m\left\{0 \leqslant t<\varepsilon:\left|X_{t}\right|>(2 t \log |\log t|)^{1 / 2}\right\} \rightarrow 0 \text { as } \varepsilon \downarrow 0 \text { a.s. }
$$

Indeed, (19) suggests that

$$
\begin{aligned}
\underset{\varepsilon \downarrow 0}{\lim \sup } \varepsilon^{-1} m\left\{0 \leqslant t<\varepsilon:\left|X_{t}\right|\right. & \left.>c(2 t \log |\log t|)^{1 / 2}\right\} \\
& =1-\exp \left\{-4\left(\frac{1}{c^{2}}-1\right)\right\} \text { a.s. }
\end{aligned}
$$


for all $0 \leqslant c \leqslant 1$. However, I have not been able to obtain even (20) via (19).

More directly connected is a result of J. Zinn and the author [5]: for a large class of processes, the function

$$
\phi(t)=q\left(\mathbf{E}\left(X_{t}-X_{0}\right)^{2}\right)^{1 / 2}, \quad 0<q<\infty,
$$

is neither an approximate upper nor lower modulus. (This class includes all Gaussian processes with stationary increments such that $\sigma(t)$ is continuous and $t^{-1} \sigma(t) \rightarrow \infty$ as $t \downarrow 0$.) For Brownian motion, slightly more is true: with probability one,

$$
\lim _{\varepsilon \downarrow 0} \varepsilon^{-1} m\left\{0 \leqslant t \leqslant \varepsilon:\left|X_{t}\right|>q \sqrt{t}\right\}
$$

does not exist for any $0<q<\infty$.

Knight [8] describes the Brownian path as having a "dense set of spine-like projections of sharpness exceeding $\sqrt{|h|}(\log 1 /|h|)^{-(1+\varepsilon)}$ for every $\varepsilon>0$," by which he means that, with probability one, there is a dense, random set $D(\omega) \in(0, \infty)$ such that for each $t \in D$ and $\varepsilon>0$,

$$
\left|X_{t+h}-X_{t}\right| \geqslant C \sqrt{|h|}(\log 1 /|h|)^{-(1+e)}
$$

for all small $h$ (depending on $\varepsilon$ ) and some constant $C$. Since Brownian motion has stationary increments, it follows from our discussion in $\$ 5$ that, for any $\gamma>0, \sqrt{h}|\log h|^{-\gamma}$ is an approximate (two-sided) lower modulus at m-a.e. point along the Brownian path. Finally, using local time methods, one can also prove (see [4]) that, for any function $p(h) \rightarrow 0$, the function $\sqrt{h}|\log h|^{-1} p(h)$ is an approximate lower modulus at every point a.s.

Appendix.

Proof of Theorem 1 for general $\Psi$. (Necessity) Define

$$
H(t)=\frac{m(\Gamma \cap(0, t))}{t}, \quad M(t)=\sup _{0<s<t} H(s), \quad t>0 .
$$

Then $H$ is continuous and we are assuming that $H(t) \rightarrow 0$ as $t \downarrow 0 ; M$ then has the same properties, as well as being nondecreasing. Let $\Psi$ be the inverse of the function $t+M(t)$. Then $\Psi$ is continuous, strictly increasing, and $\Psi(H(t))$ $<\Psi(t+M(t))=t$, which implies that (3) holds.

(Sufficiency) The proof has three main steps:

(1) Show that $H(t) \rightarrow 0$ if $H\left(\gamma^{n}\right) \rightarrow 0$ for all $0<\gamma<1$;

(2) Evaluate $\sum_{n=0}^{N} Q\left(H\left(\gamma^{n}\right)\right)$ for powers $Q(x)=x^{j}$;

(3) Approximate $\Psi$ by polynomials and use (2) to prove that

$$
\sum_{n=0}^{\infty} \int_{0}^{H\left(\gamma^{n}\right)} \Psi(s) d s \leqslant \frac{1}{1-\gamma} \int_{\Gamma} \frac{1}{t} \Psi(H(t)) d t .
$$


(1). Suppose $\left\{a_{n}\right\} \downarrow 0$ and set

$$
\beta_{n}=\int_{a_{n+1}}^{a_{n}} \frac{1}{t} I_{\Gamma}(t) d t
$$

For $t \in\left[a_{n+1}, a_{n}\right]$,

$$
\begin{aligned}
H(t) & =\frac{1}{t} \int_{0}^{a_{n+1}} I_{\Gamma}(s) d s+\frac{1}{t} \int_{a_{n+1}}^{t} I_{\Gamma}(s) d s \\
& \leqslant \frac{1}{a_{n+1}} \int_{0}^{a_{n+1}} I_{\Gamma}(s) d s+\int_{a_{n+1}}^{t} \frac{1}{s} I_{\Gamma}(s) d s \\
& \leqslant H\left(a_{n+1}\right)+\beta_{n} .
\end{aligned}
$$

Consequently,

$$
\limsup _{t \rightarrow 0} H(t) \leqslant \limsup _{n \rightarrow \infty} H\left(a_{n}\right)+\limsup _{n \rightarrow \infty} \beta_{n} .
$$

Choosing $a_{n}=\gamma^{n}, 0<\gamma<1$, we see that $\beta_{n} \leqslant \log \gamma^{-1}$ for all $n$, and hence $H(t) \rightarrow 0$ as $t \rightarrow 0$ if (and only if) $H\left(\gamma^{n}\right) \rightarrow 0$ as $n \rightarrow \infty$ for even a sequence of $\gamma$ 's approaching 1 .

(2). Fix a number $\gamma \in(0,1)$ and integers $N \geqslant 1$ and $j \geqslant 1$ :

$$
\begin{aligned}
\sum_{n=0}^{N}\left(H\left(\gamma^{n}\right)\right)^{j} & =\sum_{n=0}^{N} \int_{0}^{1} \cdots \int_{0}^{1} \gamma^{-n j} \prod_{i=1}^{j} I_{\left[0, \gamma^{n}\right]}\left(t_{i}\right) I_{\Gamma}\left(t_{i}\right) d t_{1} \cdots d t_{j} \\
& =\int_{0}^{1} \cdots \int_{0}^{1}\left(\sum_{n=0}^{N} \gamma^{-n j} \prod_{i=1}^{j} I_{\left[0, \gamma^{n}\right]}\left(t_{i}\right)\right) \prod_{i=1}^{j} I_{\Gamma}\left(t_{i}\right) d t_{1} \cdots d t_{j} \\
& =\int_{0}^{1} \cdots \int_{0}^{1}\left(\sum_{n=0}^{N \wedge Z\left(t^{*}\right)} \gamma^{-n j}\right) \prod_{i=1}^{j} I_{\Gamma}\left(t_{i}\right) d t_{1} \cdots d t_{j}
\end{aligned}
$$

where $t^{*}=\max \left(t_{1}, \ldots, t_{j}\right), N \wedge Z\left(t^{*}\right)$ stands for $\min \left(N, Z\left(t^{*}\right)\right)$, and $Z(t)=$ $\left[\log _{\gamma} t\right]$, the greatest integer less than or equal to the logarithm of $t$ to the base $\gamma$. Breaking up $[0,1\}^{j}$ into the $j$ pieces $\left\{t^{*}=t_{i}\right\}, 1 \leqslant i \leqslant j$, leads to:

$$
\begin{aligned}
\sum_{n=0}^{N}\left(H\left(\gamma^{n}\right)\right)^{j} & =j \int_{\Gamma} d t \int_{0}^{t} \cdots \int_{0}^{t} \sum_{n=0}^{N \wedge Z\left(t^{*}\right)} \gamma^{-n j} \prod_{i=1}^{j-1} I_{\Gamma}\left(t_{i}\right) d t_{1} \cdots d t_{j-1} \\
& =j \int_{\Gamma} \frac{\gamma^{-j[1+(N \wedge Z(t))]}-1}{\gamma^{-j}-1}(m(\Gamma \cap(0, t)))^{j-1} d t .
\end{aligned}
$$

(3). Let $\psi_{k}(t), 0 \leqslant t \leqslant 1$, be a sequence of polynomials which converges uniformly to $\Psi$, and define

$$
Q(t)=\int_{0}^{t} \Psi(s) d s, \quad q_{k}(t)=\int_{0}^{t} \psi_{k}(s) d s .
$$

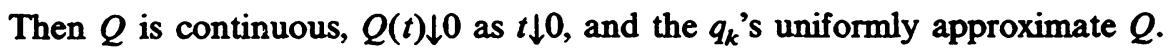
Assuming (3) and (22), we would have $Q\left(H\left(\gamma^{n}\right)\right) \rightarrow 0$ as $n \rightarrow \infty$ for every 
$\gamma \in(0,1)$, which implies that $H\left(\gamma^{n}\right) \rightarrow 0$ as $n \rightarrow \infty$ for every $\gamma \in(0,1)$. Here, then, is the proof of (22).

$$
\begin{aligned}
\sum_{n=0}^{\infty} Q\left(H\left(\gamma^{n}\right)\right) & =\lim _{N \rightarrow \infty} \sum_{n=0}^{n} Q\left(H\left(\gamma^{n}\right)\right)=\lim _{N} \sum_{n=0}^{N} \lim _{k} q_{k}\left(H\left(\gamma^{n}\right)\right) \\
& =\lim _{N} \lim _{k} \sum_{n=0}^{N} q_{k}\left(H\left(\gamma^{n}\right)\right) \\
& =\lim _{N} \lim _{k} \sum_{n=0}^{N} \sum_{j=1}^{m_{k}} a_{j}^{(k)}\left(H\left(\gamma^{n}\right)\right)^{j} \quad\left(q_{k}(t)=\sum_{j=1}^{m_{k}} a_{j}^{(k)} t^{j}\right) \\
& =\lim _{N} \lim _{k} \sum_{j=1}^{m_{k}} \sum_{n=0}^{N} a_{j}^{(k)}\left(H\left(\gamma^{n}\right)\right)^{j} .
\end{aligned}
$$

From (2),

$$
\begin{aligned}
\sum_{n=0}^{N}\left(H\left(\gamma^{n}\right)\right)^{j}= & j \int_{\Gamma} \frac{\gamma^{-j(N \wedge z(t))}}{1-\gamma^{j}}(m(\Gamma \cap(0, t)))^{j-1} d t \\
& -j \int_{\Gamma} \frac{\gamma^{j}}{1-\gamma^{j}}(m(\Gamma \cap(0, t)))^{j-1} d t .
\end{aligned}
$$

Hence,

$$
\begin{aligned}
\sum_{j=1}^{m_{k}} \sum_{n=0}^{N} a_{j}^{(k)}\left(H\left(\gamma^{n}\right)\right)^{j}= & \int_{\Gamma}\left(\gamma^{N \wedge z(t)}\right)^{-1} \sum_{j=1}^{m_{k}} j a_{j}^{(k)} \frac{\left(\lambda_{N}(t)\right)^{j-1}}{1-\gamma^{j}} d t \\
& -\int \sum_{j=1}^{m_{k}} j a_{j}^{(k)} \frac{\gamma}{1-\gamma^{j}}(\gamma m(\Gamma \cap(0, t)))^{j-1} d t,
\end{aligned}
$$

where $\lambda_{N}(t) \equiv m(\Gamma \cap(0, t)) / \gamma^{N \wedge Z(t)}$. To justify the interchange of the sum and integral needed for (23), and other operations to follow, we observe that, for all $t>0$,

$$
\begin{gathered}
0<\gamma^{N} \leqslant \gamma^{N \wedge Z(t)} ; \quad t=\gamma^{\log _{\gamma} t} \leqslant \gamma^{Z(t)}<\gamma^{N \wedge Z(t)} ; \\
\lambda_{N}(t) \leqslant H(t) \leqslant 1 .
\end{gathered}
$$

Now the sums which appear in the integrands in (23) can be expressed in terms of the $\psi_{k}$ 's as follows:

$$
\begin{aligned}
\sum_{j=1}^{m_{k}} j a_{j}^{(k)}\left(1-\gamma^{j}\right)^{-1}\left(\lambda_{N}(t)\right)^{j-1} & =\sum_{j=1}^{m_{k}} \sum_{r=0}^{\infty} j a_{j}^{(k)}\left(\gamma^{j}\right)^{r}\left(\lambda_{N}(t)\right)^{j-1} \\
& =\sum_{r=0}^{\infty} \gamma^{r} \sum_{j=1}^{m_{k}} j a_{j}^{(k)}\left(\gamma^{r} \lambda_{N}(t)\right)^{j-1} \\
& =\sum_{r=0}^{\infty} \gamma^{r} \psi_{k}\left(\gamma^{r} \lambda_{N}(t)\right)
\end{aligned}
$$


(since $\psi_{k}=q_{k}^{\prime}$ ). Similarly,

$$
\sum_{j=1}^{m_{k}} j a_{j}^{(k)} \gamma\left(1-\gamma^{j}\right)^{-1}(\gamma m(\Gamma \cap(0, t)))^{j-1}=\sum_{r=0}^{\infty} \gamma^{r+1} \psi_{k}\left(\gamma^{r+1} m(\Gamma \cap(0, t))\right) \text {. }
$$

Moreover, the integrands in (23) are bounded:

$$
\left|\left(\gamma^{N \wedge Z(t)}\right)^{-1} \sum_{r=0}^{\infty} \gamma^{r} \psi_{k}\left(\gamma^{r} \lambda_{N}(t)\right)\right| \leqslant \frac{\gamma^{-N}}{1-\gamma} \sup _{t, k}\left|\psi_{k}(t)\right|<\infty
$$

and similarly for the other one. Thus, letting $k \rightarrow \infty$,

$$
\begin{aligned}
\lim _{k} \sum_{j=1}^{m_{k}} a_{j}^{(k)} \sum_{n=0}^{N}\left(H\left(\gamma^{n}\right)\right)^{j}= & \int_{\Gamma}\left(\gamma^{N \wedge z(t)}\right)^{-1} \sum_{r=0}^{\infty} \gamma^{r} \Psi\left(\gamma^{r} \lambda_{N}(t)\right) d t \\
& -\int_{\Gamma} \sum_{r=0}^{\infty} \gamma^{r+1} \Psi\left(\gamma^{r+1} m(\Gamma \cap(0, t))\right) d t
\end{aligned}
$$

Finally, as $N \rightarrow \infty$,

$$
\left(\frac{1}{\gamma}\right)^{N \wedge Z(t)} \uparrow\left(\frac{1}{\gamma}\right)^{Z(t)}, \quad \Psi\left(\gamma^{r} \lambda_{N}(t)\right) \uparrow \Psi\left(\gamma^{r} \frac{m(\Gamma \cap(0, t))}{\gamma^{Z(t)}}\right)
$$

and hence, by the monotone convergence theorem,

$$
\begin{aligned}
\sum_{n=0}^{\infty} Q\left(H\left(\gamma^{n}\right)\right)= & \int_{\Gamma} \gamma^{-Z(t)} \sum_{r=0}^{\infty} \gamma^{r} \Psi\left(\gamma^{r} \frac{m(\Gamma \cap(0, t))}{\gamma^{Z(t)}}\right) d t \\
& -\int_{\Gamma} \sum_{r=0}^{\infty} \gamma^{r+1} \Psi\left(\gamma^{r+1} m(\Gamma \cap(0, t))\right) d t
\end{aligned}
$$

The inequality in (22) now follows because

$$
\Psi\left(\gamma^{r} \frac{m(\Gamma \cap(0, t))}{\gamma^{Z(t)}}\right) \leqslant \Psi(H(t))
$$

for all $t>0, r>0$ and $\gamma \in(0,1)$. Q.E.D.

A counterexample (see $\S 1)$. Let $0<\Lambda(t) \downarrow 0$ and $t^{-1} \Lambda(t) \uparrow+\infty$ as $t \downarrow 0$. Then there exists $a$ set $\Gamma \subset(0,1)$ such that $0 \notin \mathrm{dp}(\Gamma)$ whereas

$$
\int_{\Gamma} \frac{\Lambda(t)}{t} d t<\infty
$$

Since $\Lambda \downarrow 0$, we can choose a sequence $\left\{a_{n}\right\} \downarrow 0$ with $\Sigma \Lambda\left(a_{n}\right)<\infty$ and $2 a_{n+1}<a_{n}$ for all $n$. Define

$$
\Gamma=\bigcup_{n=1}^{\infty}\left(a_{n}, 2 a_{n}\right)
$$

Then

$$
\int_{\Gamma} \frac{\Lambda(t)}{t} d t=\sum_{n=1}^{\infty} \int_{a_{n}}^{2 a_{n}} \frac{\Lambda(t)}{t} d t<\sum_{n=1}^{\infty} \frac{\Lambda\left(a_{n}\right)}{a_{n}} \cdot a_{n}<\infty
$$


and

$$
\begin{aligned}
\underset{t \downarrow 0}{\lim \sup } H(t) & \geqslant \underset{n \rightarrow \infty}{\limsup } \frac{m\left(\Gamma \cap\left(0,2 a_{n}\right)\right)}{2 a_{n}} \\
& \geqslant \limsup _{n \rightarrow \infty} \frac{m\left(\Gamma \cap\left(a_{n}, 2 a_{n}\right)\right)}{2 a_{n}}=\frac{1}{2} .
\end{aligned}
$$

NOTE ADDED IN PROOF. Concerning the proof that $\left(3^{\prime}\right) \Rightarrow\left(2^{\prime}\right)$ for general $\Psi$ 's, Claude Dellacherie has shown me a clever reduction to the convex case. This will appear elsewhere.

\section{REFERENCES}

1. S. Berman, Local nondeterminism and local times of Gaussian processes, Indiana Univ. Math. J. 23 (1973), 69-94.

2. H. Cramer and M. R. Leadbetter, Stationary and related stochastic processes, John Wiley and Sons, New York, 1967.

3. W. Feller, An introduction to probability theory and it applications, vol. II, John Wiley and Sons, New York, 1966.

4. D. Geman and J. Horowitz, Occupation densities, Ann. Probability (to appear).

5. D. Geman and J. Zinn, On the increments of multidimensional random fields, Ann. Probability 6 (1978), 151-158.

6. C. Goffman and D. Waterman, Approximately continuous transformations, Proc. Amer. Math. Soc. 12 (1961), 116-121.

7. K. Itô and H. P. McKean, Diffusion processes and their sample paths, Academic Press, New York, 1965.

8. F. Knight, Existence of small oscillations at zeros of Brownian motion, Lecture Notes in Math., vol. 381, Springer, Berlin, 1974, pp. 134-149.

9. M. B. Marcus, Sample paths of Gaussian processes, Northwestern University, Evanston, Ill., 1977.

10. V. Strassen, An invariance principle for the low of the iterated logarithm, Z. Wahrscheinlichkeitstheorie und Verw. Gebiete 3 (1964), 211-226.

11. J. B. Walsh, Some topologies connected with Lebesgue measure, Lecture Notes in Math., vol. 191, Springer, Berlin, 1971, pp. 290-310.

Department of Mathematics, Untverstry of Massachusetts, AMherst, MassachusetTs 01003 